\title{
Low energy internal cardioversion of atrial fibrillation resistant to transthoracic shocks
}

\author{
S Mark Sopher, Francis D Murgatroyd, Alistair K B Slade, Ivan Blankoff, \\ Edward Rowland, David E Ward, A John Camm
}

\begin{abstract}
Objective-To investigate the efficacy of internal cardioversion using low energy shocks delivered with a biatrial electrode configuration in chronic atrial fibrillation resistant to transthoracic shocks.

Methods-Low energy internal cardioversion was attempted in 11 patients who had been in atrial fibrillation for 233 (SD 193) days and had failed to cardiovert with transthoracic shocks of $360 \mathrm{~J}$ in both apexbase and anterior-posterior positions. Synchronised biphasic shocks of up to $\mathbf{4 0 0}$ $\mathrm{V}(\approx 6 \mathrm{~J})$ were delivered, usually with intravenous sedation only, between high surface area electrodes in the right atrium and the left atrium (coronary sinus in nine, left pulmonary artery in one, left atrium via patent foramen ovale in one).

Results-Sinus rhythm was restored in $8 / 11$ patients. The mean leading edge voltage of successful shocks was 363 (46) V [4.9 (1.2) J]. Higher energy shocks induced transient bradycardia [time to first $R$ wave 1955 (218) ms]. No proarrhythmia or other acute complications were observed.

Conclusions-Low energy internal cardioversion of atrial fibrillation can restore sinus rhythm in patients in whom conventional transthoracic shocks have failed.
\end{abstract}

(Heart 1996;75:635-638)

Keywords: arrhythmia; atrial fibrillation; electric countershock

St George's Hospital and Medical School, Cranmer Terrace, London

S M Sopher

F D Murgatroyd

A K B Slade

I Blankoff

E Rowland

D E Ward

A J Camm

Correspondence to:

Dr M Sopher, Department

of Cardiological Sciences,

St George's Hospital

Medical School, London SW17 ORE.

Accepted for publication 27 November 1995

Transthoracic direct current capacitor discharge has been used for more than 30 years to terminate persistent atrial fibrillation. ${ }^{1}$ The restoration of sinus rhythm yields haemodynamic and symptomatic benefits and is complications associated with atrial fibrillation. However, external cardioversion using energies of up to $360 \mathrm{~J}$ fails to restore sinus rhythm in a proportion of patients. Animal studies have suggested that defibrillation efficiency can be greatly increased using biphasic shocks delivered between high surface area electrodes in an arrangement that surrounds both atria, espe- cially the right atrium-coronary sinus configuration. ${ }^{2}$ In patients, this technique is able to terminate acute atrial fibrillation with energies of 1-5 J. ${ }^{34}$ This confirms that the "biatrial" electrode configuration is a highly efficient means of electrical field delivery, and suggests that it might succeed in cases where conventional methods have failed.

We therefore attempted to terminate atrial fibrillation using low energy shocks delivered between transvenous electrodes in a biatrial configuration in a series of patients who had previously failed conventional transthoracic cardioversion.

\section{Methods}

PATIENTS

Consultants were invited to refer adult patients with persistent (more than two weeks) atrial fibrillation in whom transthoracic shocks of 360 $\mathrm{V}$, delivered between paddles in both apex-base and anteroposterior positions, had failed to restore sinus rhythm, even transiently. Patients were excluded if they had a history of stroke or peripheral embolism, or reversible cause of atrial fibrillation. All patients were studied within three months of undergoing the transthoracic shocks and a transthoracic echocardiogram.

\section{STUDY PROTOCOL}

Antiarrhythmic medication was at the discretion of the referring cardiologist, and was not interrupted for the procedure. Anticoagulation with warfarin was reduced (INR $<2 \cdot 0$ ) before the invasive procedure and subsequently restarted according to our usual practice. Intravenous sedation (midazolam and diamorphine) was given before the start of the procedure, with additional doses as required.

The procedure has been described in detail assumed to reduce the risk of thromboembolic 
Postero-anterior radiograph showing position of catheters: the defibrillation electrodes are positioned in the anterolateral right atrium $(R A)$ and coronary sinus (CS) and a pacing catheter is positioned at the apex of the right ventricle $(R V)$.

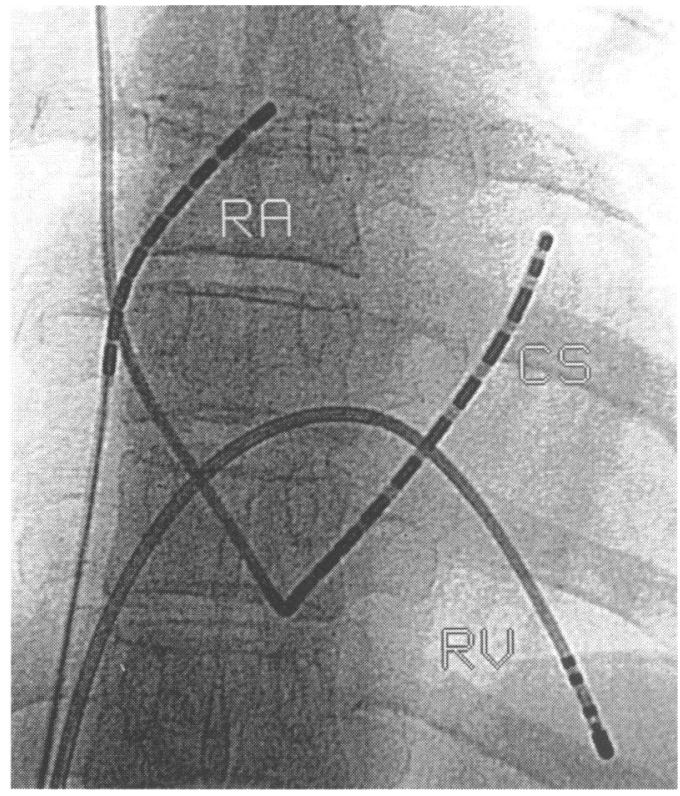

between 10 and $400 \mathrm{~V}$. To minimise the risk of ventricular proarrhythmia the device was programmed to deliver shocks only after RR intervals above $500 \mathrm{~ms}$ (see Discussion). After a test shock of $10 \mathrm{~V}$ to verify correct $\mathrm{R}$ wave synchronisation, a series of shocks at $>30 \mathrm{~s}$ intervals was given increasing from $20 \mathrm{~V}$ in $40 \mathrm{~V}$ steps until atrial fibrillation was terminated or a 400 $\mathrm{V}$ shock was delivered. If a $400 \mathrm{~V}$ shock failed to terminate atrial fibrillation the catheter positions were checked on fluoroscopy and corrected if displacement had occurred. Two further shocks of $400 \mathrm{~V}$ were then delivered. The surface electrocardiogram was recorded continuously during the procedure.

Table 1 Clinical details of the patients

\begin{tabular}{|c|c|c|c|c|c|c|}
\hline No & $\begin{array}{l}\text { Age } \\
\text { (years) }\end{array}$ & Sex & $\begin{array}{l}L A \\
\text { diameter } \\
(\mathrm{mm})\end{array}$ & $\begin{array}{l}\text { AF } \\
\text { duration } \\
\text { (days) }\end{array}$ & $\begin{array}{l}\text { Known } \\
\text { CVS } \\
\text { disease }\end{array}$ & $\begin{array}{l}A A \text { drugs } \\
\text { (at ext } \\
\text { c'version) }\end{array}$ \\
\hline 1 & 59 & $\mathrm{~F}$ & 56 & 420 & - & - \\
\hline 2 & 58 & M & 43 & 270 & - & - \\
\hline 3 & 46 & $\mathbf{M}$ & 37 & 210 & - & Amiodarone \\
\hline 4 & 48 & M & 39 & 180 & - & \\
\hline 5 & 58 & $M$ & 50 & 295 & - & Amiodarone \\
\hline 6 & 55 & $M$ & 47 & 90 & DCM (alcohol) & Verapamil \\
\hline 7 & 30 & $\mathrm{~F}$ & 56 & 50 & HOCM & Dofetilide \\
\hline 8 & 23 & $\mathbf{M}$ & 41 & 14 & DCM (idiopathic) & Quinidine \\
\hline 9 & 43 & $\mathbf{M}$ & 44 & 730 & - & \\
\hline 10 & 57 & $\mathbf{M}$ & 52 & 120 & - & Atenolol \\
\hline 11 & 69 & $\mathbf{M}$ & 49 & 180 & IHD & 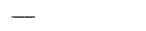 \\
\hline
\end{tabular}

$\mathrm{M}$, male; F, female; AF duration, time in continuous atrial fibrillation before internal cardioversion CVS disease, evident cardiovascular disease (ACM, alcoholic cardiomyopathy; DCM, idiopathic dilated cardiomyopathy; HOCM, hypertrophic cardiomyopathy; IHD, ischaemic heart disease); AA drugs, antiarrhythmic medication at time of failed transthoracic cardioversion (ext c'version)

Table 2 Details of internal cardioversion procedure

\begin{tabular}{|c|c|c|c|c|c|}
\hline No & $\begin{array}{l}\text { Electrode } \\
\text { configuration }\end{array}$ & $\begin{array}{l}\text { Successful } \\
\text { leading edge } \\
\text { voltage (V) }\end{array}$ & $\begin{array}{l}\text { Successful } \\
\text { energy (f) }\end{array}$ & $\begin{array}{l}\text { Impedance } \\
\text { (Ohms) } \\
\text { (at success } \\
\text { or } 400 \mathrm{~V} \text { ) }\end{array}$ & $A A$ drugs \\
\hline 1 & RA:CS & 400 & $6 \cdot 2$ & 45 & - \\
\hline 2 & RA:CS & 340 & $4 \cdot 3$ & 54 & - \\
\hline 3 & RA:CS & 360 & $4 \cdot 6$ & 64 & Amiodarone \\
\hline 4 & RA:CS & 400 & $6 \cdot 0$ & 53 & \\
\hline 5 & RA:CS & 400 & $5 \cdot 4$ & 79 & Amiodarone \\
\hline 6 & RA:LA (PFO) & 400 & $6 \cdot 2$ & 44 & \\
\hline 7 & RA:CS & 260 & $2 \cdot 5$ & 55 & Dofetilide \\
\hline 8 & RA:CS & 400 & $4 \cdot 1$ & 70 & - \\
\hline 9 & RA:CS & - & - & 68 & - \\
\hline 10 & RA:CS & - & - & 64 & - \\
\hline 11 & RA:LPA & - & - & 56 & - \\
\hline
\end{tabular}

RA, right atrium; CS, coronary sinus; LA (via PFO), left atrium through patent foramen ovale LPA, left pulmonary artery; AA drugs, antiarrhythmic medication at time of procedure.
The invasive protocol was approved by the local research ethics committee, and all patients gave written informed consent.

\section{Results}

PATIENTS

Eleven patients entered the study (table 1). The mean duration of atrial fibrillation at the time of the procedure was 233 (SD 193) days. Four patients had evidence of underlying cardiac disease. Six patients had been taking an antiarrhythmic drug at the time of the attempt at external cardioversion but this had been discontinued in three before referral for internal cardioversion.

\section{OUTCOMES}

There were four deviations from protocol. In two early patients, the procedure was performed under general anaesthesia in anticipation of the need for high energy internal cardioversion. In two patients, the coronary sinus could not be cannulated: the left atrial electrode was positioned in the left main pulmonary artery in one and in the left atrium itself (through a patent foramen ovale) in the other.

Successful cardioversion (defined as the immediate return of sinus rhythm following a shock) was achieved in eight patients. The mean (SD) leading edge voltage of successful shocks was 363 (46) V (range, 260-400 V) and the mean energy was $4.9(1 \cdot 2) \mathrm{J}$ (range, $2 \cdot 5$ $6 \cdot 2 \mathrm{~J}$ ).

Three patients failed to cardiovert despite three shocks of $400 \mathrm{~V}$.

\section{COMPLICATIONS}

No ventricular arrhythmia was seen acutely following a shock. Bradycardia was seen following higher energy shocks whether or not sinus rhythm was restored. The mean of the longest delay from any shock to the first $\mathrm{R}$ wave in each patient was 1955 (218) ms (range, 400-2640 $\mathrm{ms})$. However, no patient required back up ventricular pacing as this bradycardia was transient.

Two patients experienced possible late complications of the technique of internal cardioversion or of the reduction in the level of their anticoagulation. Patient 2 presented locally five days after the successful cardioversion with a four day history of an increasingly cold and numb right lower limb. He had reverted to atrial fibrillation 24 hours after the procedure. An embolus to the right popliteal artery was confirmed and successfully treated. Patient 9 collapsed out of hospital eight hours after the unsuccessful but apparently uncomplicated procedure. Cardiopulmonary resuscitation was only instituted around 15 minutes later when ventricular fibrillation was recorded. Following a prolonged resuscitation he suffered severe hypoxic cerebral damage and died after three weeks. No cerebral infarct or source of embolus was identified. There had been no history suggestive of ischaemic heart disease and there was no clear evidence of acute myocardial infarction. However, necropsy examination revealed coronary atheroma particularly involv- 
ing the left coronary artery and the suggestion of a small recent ventricular septal infarct. The relation, if any, of this to the procedure is unclear.

\section{Discussion}

CARDIOVERSION OF ATRIAL FIBRILLATION

Atrial fibrillation is the commonest sustained cardiac arrhythmia and accounts for the most hospital admissions and days in hospital. ${ }^{5}$ It frequently causes disabling symptoms and haemodynamic impairment and is an important treatable cause of stroke. ${ }^{6}$ Restoration of sinus rhythm results in improved left ventricular function and functional capacity even in patients with a previously controlled ventricular rate..$^{7-9}$ Electrical cardioversion is more effective than using antiarrhythmic drugs and has become the standard technique for cardioversion of chronic atrial fibrillation. However, the technique is not uniformly effective. The age of the patient and the arrhythmia duration have been shown to be independent predictors of failure, ${ }^{10}$ while other factors such as left atrial diameter and rheumatic aetiology may also be used to indicate a likelihood of failure. ${ }^{11}$

Patients in whom transthoracic cardioversion fails are generally accepted as being in permanent atrial fibrillation. However, other methods can be used to obtain the benefits of sinus rhythm. Antiarrhythmic drugs alone are rarely effective in cardioverting chronic atrial fibrillation resistant to transthoracic shock, but an increased success rate can be achieved when cardioversion is repeated after loading with amiodarone. ${ }^{12}$ In contrast, class Ic antiarrhythmic drugs may increase the atrial defibrillation threshold. ${ }^{13}$ Surgical options such as the maze operation $^{14}$ may restore and maintain sinus rhythm but are major procedures with significant morbidity. Simpler operations which do not restore mechanical function of the atria, such as left atrial isolation and the corridor procedure, ${ }^{15}$ may not achieve the haemodynamic benefit of normal sinus rhythm or reduce the thromboembolic risk associated with atrial fibrillation.

\section{INTERNAL CARDIOVERSION}

Another approach is to try to increase the efficacy of electrical cardioversion by using an internal electrode arrangement that increases the potential gradient achieved within atrial tissue. Low energy intracardiac shocks have been known to terminate ventricular fibrillation refractory to multiple transthoracic shocks, ${ }^{16}$ but have only recently been investigated in atrial fibrillation. Capacitor discharge between the proximal pole of a pacing catheter in the right atrium and a backplate has been described by Lévy et al. ${ }^{17} \mathrm{~A}$ higher success rate was obtained with this method than with repeated external cardioversion (91\% v67\%) in a randomised trial of patients with "resistant" atrial fibrillation after pretreatment with amiodarone. ${ }^{12}$ However this technique uses $200-300$ $\mathrm{J}$ intracavitary shocks, which are known to cause arcing with gas bubble formation, which can result in barotrauma. ${ }^{18}$ This may account for severe complications recently reported, ${ }^{19}$ and may be responsible for reluctance to use the technique.

In theory, the most efficient electrode configuration for atrial defibrillation is in proximity to the atria and providing a field which embraces both atria. A systematic investigation in a sheep model of acute atrial fibrillation found that a defibrillation threshold of around $1 \mathrm{~J}$ could be obtained with a biphasic shock waveform and a biatrial high surface area electrode configuration, especially one using the coronary sinus for the left sided electrode. ${ }^{2}$ The efficacy of this method has been confirmed in patients with induced and spontaneous paroxysmal atrial fibrillation. ${ }^{4}$ The atrial defibrillation threshold was generally $1-5 \mathrm{~J}$. General anaesthesia is therefore unnecessary. The method has been effective in two patients in whom morbid obesity might account for failure of transthoracic cardioversion. ${ }^{20}$ However, the technique has not previously been applied systematically to a series of patients with persistent atrial fibrillation refractory to conventional transthoracic shocks.

In our study, biphasic biatrial shocks terminated atrial fibrillation in eight out of 11 patients who had previously failed external cardioversion. No formal attempt was made to select patients with a high likelihood of success. However, as in previous studies, patients were most likely to be referred for cardioversion if it was perceived that there was a reasonable chance of success, or a particular reason for the patient to benefit from sinus rhythm. Thus the patients were young (23 to 69 years) compared to the population with chronic atrial fibrillation; none had significant valvar heart disease; and while most had left atrial dilatation, this did not exceed $5.6 \mathrm{~cm}$. None of the patients studied were obese or had chest hyperexpansion which might have affected the transthoracic impedance. Those that were taking antiarrhythmic drug treatment had previously failed external cardioversion while on the same treatment.

With a limited number of subjects, it is not possible to draw firm conclusions about why the technique failed in three patients. One (No 10) was the first patient with "resistant" atrial fibrillation to undergo the technique, and only one attempt was made at maximum output $(400 \mathrm{~V})$. In patient 11 , the left sided electrode was situated in the left main pulmonary artery as the coronary sinus could not be cannulated. This position is associated with a higher defibrillation threshold in the sheep model..$^{2}$ Patient 9 had been in atrial fibrillation for considerably longer than others in the series. In all cases, the energy delivered (up to $6 \cdot 2 \mathrm{~J}$ ) was limited by the maximum output $(400 \mathrm{~V})$ of the defibrillator used. It is not known whether a higher output would have further increased the likelihood of success.

The study did not address the issue of maintenance of sinus rhythm following successful cardioversion and there was no policy regarding the use of antiarrhythmic drugs. In most series, the risk of atrial fibrillation recurrence within a year of cardioversion is approximately $50 \%$, and this is likely to be higher in patients with "resistant" atrial fibrillation. ${ }^{102122}$ However, 
antiarrhythmic treatment can considerably reduce the risk of atrial fibrillation recurrence $^{2223}$ and should therefore be considered routinely for patients undergoing this technique.

\section{SAFETY}

The risk that biatrial shocks may induce ventricular tachyarrhythmias has been systematically evaluated in sheep. ${ }^{24}$ Such proarrhythmia does not occur if shocks are synchronised to the $R$ wave and delivered following an $R R$ interval of more than $300 \mathrm{~ms}$. To allow a margin of safety, we programmed the defibrillator to a minimum interval of $500 \mathrm{~ms}$. In a rapidly growing worldwide experience, no proarrhythmia has been seen with correctly synchronised shocks delivered in patients using a biatrial configuration. ${ }^{25}$

Intracavitary shocks delivered from point electrodes can cause microscopic changes of subendocardial necrosis in the right atrium, ${ }^{26} 27$ and serious complications have been reported for shocks of more than $100 \mathrm{~J}$ delivered within the coronary sinus in animals ${ }^{2829}$ and man. ${ }^{30}$ However, no injury has been reported with shocks of $30 \mathrm{~J}$ in current ventricular defibrillation systems employing electrodes in the coronary sinus. ${ }^{31}{ }^{32}$ This is presumably because, with high surface area electrodes, defibrillation relies solely on the electrical field generated and not on barotrauma.

There were no acute arrhythmic or mechanical complications in the present study. However marked transient bradycardia was common following shocks above $4 \mathrm{~J}$. Pacing was not necessary, but it would seem a prudent precaution to have this facility in patients with longstanding atrial fibrillation whose sinus node function is undetermined. Precautions to reduce the risks of excessive bleeding or thromboembolic complications are also required when performing an invasive procedure in patients anticoagulated for atrial fibrillation.

\section{CONCLUSIONS}

Sinus rhythm can be restored in a proportion of patients with atrial fibrillation in whom conventional transthoracic shocks have failed, using biphasic shocks delivered between the right atrium and the coronary sinus. The energy required is very low, and general anaesthesia is not required. Low energy internal cardioversion should be considered in patients for whom conventional methods have failed or are impossible. The role of this new technique should be formally explored in large scale studies.

SMS is supported by a Junior Research Fellowship of the British Heart Foundation. This study was funded in part by grants from the British Heart Foundation and from InControl, Inc.

1 Lown B, Amarasingham R, Neuman J. New method for terminating cardiac arrhythmias-use of synchronized capacminating cardiac arrhythmias-use of syn
itor discharge. $¥ A M A$
$1962 ; 182: 548-55$.

2 Cooper RA, Alferness CA, Smith WM, Ideker RE. Internal cardioversion of atrial fibrillation in sheep. Circulation cardioversion of

3 Keane D, Boyd E, Anderson D, et al. Comparison of biphasic and monophasic waveforms in epicardial atrial defibrilla and monophasic waveforms in epicardial

4 Murgatroyd FD, Slade AKB, Sopher SM, Rowland E, Ward DE, Camm AJ. Efficacy and tolerability of transvenous low energy cardioversion of paroxysmal atrial fibrillation in humans. F Am Coll Cardiol 1995;25:1347-53.

5 Bialy D, Lehmann MH, Schumacher DN, Steinman RT, Meissner MD. Hospitalization for arrhythmias in the United States: importance of atrial fibrillation [abstr]. $\mathcal{F}$ Am Coll Cardiol 1992;19:41A.

6 Anonymous. Stroke Prevention in Atrial Fibrillation Study. Final results. Circulation 1991;84:527-39.

7 Kubac G, Malowany L. Functional capacity of patients with atrial fibrillation and controlled heart rate before and after cardioversion. Can $\mathcal{F}$ Cardiol 1992;8:941-6.

8 Alam $M$, Thorstrand C. Left ventricular function in patients with atrial fibrillation before and after cardioversion. $A m \mathcal{J}$ Cardiol 1992;69:694-6.

9 Gosselink ATM, Crijns HJ, Van Den Berg MP, et al. Functional capacity before and after cardioversion of atrial fibrillation: a controlled study. Br Heart $₹$ 1994;72:161-6.

10 Van Gelder IC, Crijns HJ, Van Gilst WH, Verwer R, Lie KI. Prediction of uneventful cardioversion and maintenance of sinus rhythm from direct-current electrical cardioversion of chronic atrial fibrillation and flutter. Am $\mathcal{F}$ Cardiol 1991; 68:41-6.

11 Lown B. Electrical reversion of cardiac arrhythmias. $\mathrm{Br}$ Heart F 1967;29:469-89.

12 Levy S, Lauribe P, Dolla E, et al. A randomized comparison of external and internal cardioversion of chronic atrial fibrillation. Circulation 1992;86:1415-20.

13 Van Gelder IC, Crijns HJGM, Van Gilst WH, DeLangen CDJ, Van Wijk LM, Lie KI. Effects of flecainide on the atrial defibrillation threshold. Am f Cardiol 1989;63: $112-4$.

14 Cox JL, Boineau JP, Schuessler RB, et al. Successful surgical treatment of atrial fibrillation. Review and clinical update. fAMA 1991;266:1976-80.

15 Leitch JW, Klein GJ, Yee R, Guiraudon GM. Sinus nodeatrioventricular node isolation: long-term results with the "corridor" operation for atrial fibrillation. $\mathcal{f} \mathrm{Am}$ Coll Cardiol 1991;17:970-5.

16 Cohen TJ, Scheinman MM, Pullen PT, et al. Emergency intracardiac defibrillation for refractory ventricular fibrillation during routine electrophysiological study. $\mathcal{F ~} \mathrm{Am} \mathrm{Coll}$ Cardiol 1991;18:1280-4.

17 Levy S, Lacombe P, Cointe R, Bru P. High energy transcatheter cardioversion of chronic atrial fibrillation. $₹ \mathrm{Am}$ Coll Cardiol 1988;12:514-8.

18 Bardy GH, Coltorti F, Stewart RB, Greene HL, Ivey TD. Catheter-mediated electrical ablation: the relation between current and pulse width on voltage breakdown and shockwave generation. Circ Res 1988;63:409-14.

19 Blanc J, Larlet J, Salaun G, Maheu B, Mansourati J. Is right atrial intracavitary direct-current shock for atrial fibrillaatrial intracavitary direct-current shock
tion safe? [abstr]. PACE 1995;18:851.

20 Baker BM, Botteron GW, Smith JM. Low-energy internal cardioversion for atrial fibrillation resistant to external carcardioversion for atrial fibrillation resistant to extern
dioversion. $\mathcal{F}$ Cardiovasc Electrophysiol 1995;6:44-7.

21 Brodsky MA, Allen BJ, Capparelli EV, Luckett CR, Morton R, Henry WL. Factors determining maintenance of sinus $\mathrm{R}$, Henry WL. Factors determining maintenance of sinus
rhythm after chronic atrial fibrillation with left atrial dilatathythm after chronic atrial fibrillation

22 Coplen SE, Antman EM, Berlin JA, Hewitt P, Chalmers TC. Efficacy and safety of quinidine therapy for maintenance of sinus rhythm after cardioversion. A meta-analysis of randomized control trials. Circulation 1990;82:1106-16.

23 Crijns HJ, Van Gelder IC, Van Gilst WH, Hillege $H$, Gosselink ATM, Lie KI. Serial antiarrhythmic drug treatment to maintain sinus rhythm after electrical cardioversion for chronic atrial fibrillation or atrial flutter. $A m \mathcal{F}$ Cardiol 1991;68:335-41.

24 Ayers GM, Alferness CA, Ilina $M$, et al. Ventricular proarrhythmic effects of ventricular cycle length and shock strength in a sheep model of transvenous atrial defibrillation. Circulation 1994;89:413-22.

25 Murgatroyd FD, Johnson EE, Cooper RA, et al. Safety of low energy transvenous atrial defibrillation-world experience [abstr]. Circulation 1994;90:14.

26 Dunbar DN, Tobler HG, Fetter J, Gornick CC, Benson DWJ, Benditt DG. Intracavitary electrode catheter cardioversion of atrial tachyarrhythmias in the dog. $7 \mathrm{Am} \mathrm{Col}$ Cardiol 1986;7:1015-27.

27 Kumagai K, Yamanouchi Y, Tashiro N, Hiroki T, Arakawa $\mathrm{K}$. Low energy synchronous transcatheter cardioversion of atrial flutter/fibrillation in the dog. $\mathrm{f} \mathrm{Am}$ Coll Cardio 1990;16:497-501.

28 Coltorti F, Bardy GH, Reichenbach D, et al. Catheter-mediated electrical ablation of the posterior septum via the coronary sinus: electrophysiologic and histologic observacoronary sinus: electrophysiologic and his
tions in dogs. Circulation 1985;72:612-22.

29 Coltorti F, Bardy GH, Reichenbach D, et al. Effects of varying electrode configuration with catheter-mediated defibing electrode configuration with catheter-mediated defib-
rillator pulses at the coronary sinus orifice in dogs. rillator pulses at the coronar
Circulation 1986;73:1321-33.

30 Fisher JD, Brodman R, Kim SG, Matos JA, Wallerson D, Waspe LE. Attempted nonsurgical electrical ablation of accessory pathways via the coronary sinus in the WolffParkinson-White syndrome. $₹ \mathrm{Am}$ Coll Cardiol 1984;4: 685-94.

31 Bardy GH, Allen MD, Mehra R, Johnson G. An effective and adaptable transvenous defibrillation system using the and adaptable transvenous defibrillation system using the 887-95.

32 Bardy GH, Allen MD, Mehra R, et al. Transvenous defibrillation in humans via the coronary sinus. Circulation 1990 ; lation in humar
81:1252-9. 